\title{
DESAFÍOS ÉTICOS DE LA MANIPULACIÓN GENÉTICA Y LA INVESTIGACIÓN CON ANIMALES
}

\section{ETHICAL CHALLENGES OF GENETIC MANIPULATION AND RESEARCH WITH ANIMALS}

Eduardo Rodríguez Yunta ${ }^{1, a}$

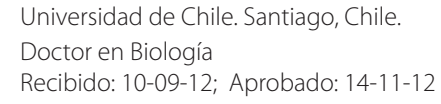

\begin{abstract}
RESUMEN
En la investigación con animales existen cuestionamientos éticos tanto en su uso como modelos de enfermedades humanas y el ser requisito previo para ensayos en humanos, como en la introducción de modificaciones genéticas. Algunos de estos cuestionamientos se refieren a que no representan completamente la condición humana como modelo; que realizar pruebas de toxicidad causan grave daño a los animales; que se altera su naturaleza mediante modificaciones genéticas y el riesgo de introducir organismos genéticamente modificados. El uso de animales en investigación para beneficio humano impone la responsabilidad moral de respetarlo, no haciéndoles sufrir innecesariamente, puesto que se está trabajando con seres vivos con capacidad de sentir.
\end{abstract}

Palabras clave: Bioética; Experimentación animal; Ensayos clínicos como asunto; Animales modificados genéticamente (fuente: DeCS BIREME).

\begin{abstract}
Research with animals presents ethical questions both for being used as models of human diseases and for being a prerequisite for trials in humans, as in the introduction of genetic modifications. Some of these questions refer to the fact that, as models, they do not fully represent the human condition; that conducting toxicity tests causes great harm to animals; that their nature is altered by genetic modifications and that introducing genetically modified organisms is a risk. The use of animals in research for the benefit of humans imposes the moral responsibility to respect them, not making them suffer unnecessarily, since they are living beings capable of feeling.
\end{abstract}

Key words: Bioethics; Animal experimentation; Clinical trials as topic; Animals, genetically modified (source: MeSH NLM).

\section{INTRODUCCIÓN}

Los códigos de ética internacionales para la investigación biomédica consideran una obligación realizar ensayos con animales antes de efectuarlos en seres humanos. Según el Código de Núremberg cualquier experimento realizado en seres humanos debe ser diseñado y basado en los resultados de investigación animal. La Declaración de Helsinki considera que la investigación clínica en seres humanos debe estar basada en pruebas de laboratorio adecuadamente realizadas y en experimentación con animales.

A la fecha, no existen alternativas viables para no experimentar con animales. Las técnicas de cultivo celulares y las simulaciones computacionales solo proporcionan datos accesorios útiles y lo máximo que pueden servir es para disminuir el número de animales que se han de utilizar para experimentación. Investigar en animales para extrapolar datos en humanos se justifica debido a las similitudes biológicas a nivel fisiológico, aunque el último dato debe realizarse siempre en humanos. Así, para que una agencia reguladora apruebe un nuevo fármaco en el mercado se debe pasar de una fase preclínica de investigación con cultivos celulares y animales a tres fases sucesivas de ensayos clínicos en humanos. El animal de experimentación constituye un buen modelo para evaluar el efecto de un producto químico, pues en él se examina el potencial tóxico, las vías de acceso, la excreción y el metabolismo. Sin embargo, la más sofisticada tecnología no puede imitar las complicadas interacciones entre células, tejidos y órganos que se dan en un cuerpo humano.

Por otra parte, muchas de las investigaciones actuales con animales tienen que ver con el desarrollo de la biotecnología y la posibilidad de patentar formas de vida. Con el advenimiento de la ingeniería genética se dispone de una tecnología que permite rediseñar organismos adaptados de forma rápida y controlada al intervenir el genoma. La biotecnología es capaz de superar las restricciones que existen entre especies, rompe límites que a lo largo de los siglos se han dado

Citar como: Rodríguez Yunta E. Desafíos éticos de la manipulación genética y la investigación con animales. Rev Peru Med Exp Salud Publica. 2012;29(4):535-40. 
por imposibilidad de apareamiento, pudiéndose referirse en la actualidad a la tecnificación del ser vivo.

En el presente artículo se exponen los cuidados necesarios a considerar con los animales de experimentación como paso previo a los ensayos clínicos en humanos y reflexiona desde la bioética el uso del animal como modelo y la eticidad de introducir modificaciones genéticas en animales mediante ingeniería genética.

\section{MODELOS ANIMALES EN INVESTIGACIÓN BIOMÉDICA}

Las ventajas de los modelos animales radican en ser sistemas más simples que el humano. Se pueden aislar acciones específicas, los tiempos de generación y ciclos de vida son más cortos y se pueden realizar procedimientos difíciles de completar en humanos.

El desarrollo de una nueva medicina es un proceso largo y complejo. Para cumplir con los requisitos de las regulaciones se debe demostrar que un procedimiento $o$ medicina potencial posee un nivel aceptable de seguridad en animales y entonces medir la biodisponibilidad (1). La evaluación de la seguridad se realiza mediante el análisis de toxicidad en órganos y tejidos, con énfasis en el aparato reproductor si más aun se tiene la intención de administrar el producto posteriormente en mujeres en edad reproductiva ${ }^{(2)}$.

En pruebas de toxicidad hay que tener en cuenta:

- La inducción de efectos adversos es intencional, para evaluar los límites de seguridad.

- Las pruebas son un requisito legal.

- La armonización de requisitos internacionales para pruebas de toxicidad puede evitar duplicaciones y daños innecesarios para los animales.

- Se induce toxicidad extra en el animal, con el fin de asegurar que la carencia de efectos no sea debida a defectos en la metodología. Se calcula la dosis letal media DL50 que mata al 50\% de los animales.

- El daño a los animales es parte integral de la prueba y se considera inevitable.

- Generalmente se practica eutanasia en el animal al final del experimento.

Los efectos toxicológicos a estudiarse incluyen la inducción de mutaciones, malformaciones en el desarrollo (teratogénesis), la posibilidad de inducción de cáncer (carcinogénesis) y efectos en la fertilidad.

Los estudios de biodisponibilidad incluyen: 1) la medida de absorción que estudia las rutas de administración, dosis, efectos de la ingesta alimenticia, porcentaje de absorción y el efecto del paso por el hígado. 2) La distribución en los tejidos, la acumulación en el suero, el líquido cefalorraquídeo, orina y bilis. 3) El metabolismo. 4) La excreción en cantidades y rutas.

Los resultados de pruebas en animales se usan para decidir si los probables efectos beneficiosos del procedimiento a estudiar superan los riesgos de efectos secundarios adversos tóxicos y para establecer una dosis segura de uso en ensayos clínicos con seres humanos. Indican posibles efectos adversos que deben monitorearse cuidadosamente.

\section{CUIDADO DE LOS ANIMALES DE EXPERIMENTACIÓN}

La fase de estudios en animales requiere procedimientos estandarizados a nivel internacional, lo cual exige tener bioterios acreditados. Un bioterio es el lugar físico donde se alojan, crían y utilizan animales de laboratorio (el animal de experimentación más usado es el ratón). Estos deben mantener una calidad genética y microbiológica definida y un ambiente estandarizado de acuerdo con la especie, variedad y raza, y sus características de sexo, edad, tamaño, conducta y salud. Se han de crear condiciones de aislamiento ambiental con personal entrenado y calificado para evitar infecciones y contaminaciones microbianas. También se ha de mantener una pureza genética mediante la regulación de apareamientos para evitar variabilidad no controlada y así disminuir el número necesario de animales que se necesita. Mantener un bioterio es costoso, pero el no tenerlo con las condiciones adecuadas hace que las investigaciones que se realicen no sean válidas, ya que habría muchas variables no controladas. Por otra parte, los costos de tener que repetir investigaciones no válidas son mucho mayores.

Para el bienestar animal, hay que tener en consideración el diseño experimental (3):

- La habilidad de los animales para integrar grupos con sus semejantes, a través de la vista, olfato y posible contacto, ya sea que los animales se mantengan aislados o en grupos.

- El diseño y construcción del alojamiento, evitando la presencia de materiales peligrosos o que causen enfermedad.

- La disponibilidad y adecuación de elementos que enriquezcan el medio ambiente.

- Las metas del proyecto y el diseño experimental (producción, crianza, investigación, pruebas de laboratorio y educación).

- La intensidad de la manipulación animal y el grado de daño que causen los procedimientos.

- La duración del período de permanencia de los animales.

Dentro del bioterio se encuentran los encierros primarios donde se confinan los animales aislados del resto, 
que deben satisfacer condiciones adecuadas para el bienestar animal (Tabla 1).

Los factores genéticos se deben controlar para que se mantenga el genotipo; el bioterio y el encierro primario deben aislarse para que no haya contaminación microbiológica ni de otros animales (Tabla 2). También es necesario mantener condiciones de seguridad para evitar zoonosis (enfermedad transmisible del animal al hombre) y alergias ${ }^{(4)}$.

Las Pautas Éticas Internacionales del Consejo Internacional de Organizaciones Médicas (CIOM) para Investigación Biomédica con Animales señalan los criterios éticos que deben seguirse en el manejo y cuidado de animales para experimentación. Tienen su base en el principio de las $3 R$ formulado por William Russell y Rex Burch en 1959 con antecedente en el trabajo de Marshall Hall de $1831^{(5,6)}$. Las $3 R$ significan reemplazo, reducción y refinamiento.

1) Reemplazo de animales conscientes por animales inconscientes o materiales no sensibles. Son alternativas de reemplazo: uso de técnicas físicas y

Tabla 1. Condiciones adecuadas de un encierro primario.

Necesidades fisiológicas y de conducta
Procurar la correcta eliminación de los desechos productos de
la micción y defecación de los animales de experimentación.
Cuidar la adecuada limpieza, humedad y ventilación del am-
biente. Mantenimiento de la temperatura corporal. Movimien-
tos normales y postura. Cama o suelo donde descansa el ani-
mal libre de polvo, hecha de material no tratado químicamente,
poder de absorción adecuado, con recambio a intervalos. Ac-
ceso a agua y alimento. Reproducción (si está indicado).
Interacciones sociales
Entre individuos de la misma especie y el establecimiento
de jerarquía, evitando la sobrepoblación.

\section{Seguridad}

Tener un medio ambiente seguro, que impida el escape de los animales o que se entrampen entre superficies opuestas o en aberturas estructurales.

Ausencia de bordes cortantes o proyecciones que puedan causar lesiones.

Permitir la observación de los animales con la mínima molestia para ellos.

Condiciones ambientales

Control de la temperatura, humedad, aireación, presión de aire y gases mediante un sistema de climatización con diseño apropiado basado en la producción de calor de los animales y ajuste automático de los valores preseleccionados.

Control de la luz (periodo, intensidad, color) mediante un encendido periódico automático, la reducción gradual de luz, la intensidad de rayos ultravioleta.

Control de sonido (ruido, intensidad, frecuencia): lograr aislamiento para evitar ruidos, prevención del sonido originado durante el trabajo, insonorización del equipo y las instalaciones.

Alimento

Formulación adecuada con ingredientes naturales, dieta purificada y definida químicamente, control de calidad.
Tabla 2. Control del genotipo y contaminación microbiológica.

\begin{tabular}{l} 
Control de genotipo \\
Mediante endogamia, exogamia, esquemas de rotación o \\
esquemas de reproducción asistida por ordenador. \\
Control de endoflora y parásitos \\
Lograr que los animales experimentales sean gnotobióticos \\
(ausencia de patógenos) o SPF (libre de gérmenes patógenos). \\
Aislamiento de gérmenes ambientales \\
Mediante una barrera higiénica efectiva y un apropiado diseño \\
de instalaciones de aire y su renovación (filtros de aire, filtros \\
de polvo, filtros de aire particulado de alta eficacia HEPA). \\
Descontaminar alimento, agua y cama mediante pasteuriza- \\
ción, esterilización, irradiación o filtración, con procedimien- \\
tos normalizados de trabajo definidos. \\
Descontaminación de personal mediante Air-lock de paso \\
para evitar entrada de aire externo, cambio de ropa e higiene. \\
Aislamiento de animales \\
Ausencia de insectos mediante barreras de diseño apropia- \\
do de las instalaciones. \\
Ausencia de roedores salvajes mediante cuarentena, barre- \\
ras y medidas higiénicas de contención efectiva. \\
\hline SPF: Specific Pathogens Free; HEPA: High-Efficiency Particulate Air.
\end{tabular}

químicas y predicciones basadas en las propiedades físicas de las moléculas, uso de modelos matemáticos y de computación, uso de organismos inferiores como invertebrados o microorganismos, uso de organismos muertos, uso de estados primarios de desarrollo, uso de métodos in vitro (fracciones subcelulares, fracciones de tejidos, suspensiones celulares, órganos, cultivos celulares, incluyendo células humanas).

2) Reducción del número de animales sin disminución de la precisión. Esto se logra con colonias genéticamente homogéneas, sin influencias ambientales, seleccionando el modelo animal adecuado, usando una metodología bioestadística avanzada y un banco de datos adecuado en que se publica tanto los resultados positivos como los negativos para no repetir experimentos.

3) Refinamiento de las técnicas para reducir el dolor y las molestias. Se debe considerar los siguientes aspectos:

- Cuidado y bienestar animal para evitar molestias innecesarias.

- Destrezas y capacitación del personal para dar el tratamiento adecuado a los animales de experimentación.

- Perfeccionamiento de métodos para detectar dolor.

- Uso de anestésicos, analgésicos y tranquilizantes para disminuir el dolor.

- Uso de técnicas no invasivas o telemétricas para evitar dolor y molestias.

- Uso de radiografía (tomografías) para detectar tumores o deterioro orgánico interior.

- Aplicar eutanasia anticipada o finalización del procedimiento doloroso (llamado punto final) para evitar la prolongación de sufrimiento. 


\section{ANESTESIA Y EUTANASIA EN ANIMALES}

Los objetivos de la anestesia son:

- Facilitar la manipulación del animal o la realización de procedimientos quirúrgicos o dolorosos.

- Proporcionar un trato humanitario a los animales, reduciendo al mínimo el sufrimiento asociado a dicha manipulación y evitando situaciones dolorosas, de angustia o ansiedad.

- Reducir al mínimo las consecuencias negativas de la cirugía sobre la fisiología del animal.

- Evaluar si se permite la realización de investigaciones que no podrían realizarse con el animal consciente.

Es importante tener en cuenta: 1) Determinar la especie del animal, su estado actual, el objetivo del experimento, tipo de procedimiento, duración, experiencia del investigador y los medios con los que se dispone. 2) Los anestésicos generales producen una parálisis descendente no selectiva del sistema nervioso central por lo que hay que calcular la dosis para no producir daños. Se debe tener cuidado de no producir depresión respiratoria. 3) La anestesia debe realizarla personal debidamente entrenado.

Con relación a la eutanasia en animales, esta se define como la destrucción humanitaria realizada por un método que produzca inconsciencia rápida y la muerte subsiguiente sin evidencia de dolor o molestia, o un método que utilice anestesia producida por un agente que cause pérdida de conciencia indolora y muerte subsiguiente ${ }^{(7)}$. Se requiere mínima inmovilización, evitar excitación, ha de ser apropiado, minimizando el miedo y el estrés en el animal, fiable, reproducible, irreversible, sencillo de administrar y seguro para el operador.

La eutanasia se puede aplicar cuando se producen efectos adversos prolongados para el animal luego del experimento, provocando un grado de sufrimiento superior al previsto o, cuando se tiene que realizar un sangrado total u obtener tejidos para un estudio. En la elección del método de eutanasia es importante elegirlo de acuerdo a la especie, edad y salud del animal por lo que se recomienda consultar con un veterinario.

\section{MODIFICACIONES GENÉTICAS EN ANIMALES EXPERIMENTALES}

Mediante la ingeniería genética se pueden introducir genes de una especie a otra. Los organismos genéticamente modificados denominados también transgénicos son aquellos cuyo genoma tiene un gen añadido o alterado en sus células, incluyendo las células germinales. La generación de nuevos organismos con introducción de propiedades ha permitido patentar organismos vivos, siempre que tengan utilidad industrial, lo que ha posibilitado el crecimiento enorme de la biotecnología y generado intereses comerciales. La directiva europea de la oficina de patentes define un organismo genéticamente modificado o transgénico como aquel cuyo material genético ha sido modificado de una manera que no se produce naturalmente en el apareamiento ni en la recombinación natural ${ }^{(8)}$. La transferencia de genes de una especie a otra se realiza con el propósito de que el ácido desoxirribonucleico (ADN) externo contribuya a la síntesis de la proteína que codifica en el organismo huésped. Estos animales se fabrican usando una construcción transgénica con la secuencia del gen que se piensa introducir.

Existen diferentes técnicas de incorporación de ADN externo en animales, la más común consiste en la inyección de transgenes (genes transferidos) en el pronúcleo de un cigoto ${ }^{(9)}$. La integración es aleatoria en cromosomas del huésped y la expresión de los transgenes depende de la función de los sitios de integración. Normalmente, la transferencia del genoma lleva consigo construcciones de genes combinadas artificialmente con fragmentos de ADN consistentes en secuencias reguladoras de codificación de proteínas. Se pueden inyectar múltiples fragmentos de ADN para aumentar la eficacia de inserción y actualmente también se insertan cromosomas artificiales. Los cigotos o embriones son transferidos a hembras receptoras y los animales nacidos son examinados para comprobar si los genes inyectados se han incorporado a la cadena de ADN. Aunque esta técnica es fiable, tiene en general baja eficacia.

También se usa como método el transferir genes dirigidos hacia un blanco en un gen huésped, lo que tiene interés en técnicas de terapia reparativa. El gen de interés se modifica en células del tronco embrionario mediante recombinación homóloga usando un vector que es una versión modificada del gen huésped ${ }^{(10)}$. Estas células después se inyectan a un blastocisto para obtener animales quiméricos (en los que solo algunas células portan el gen transferido). Después se cruzan los animales quiméricos y se obtienen ratones transformados con el gen transferido en todas las células.

Otras técnicas consisten en la producción de transgénicos usando como vectores retrovirus (se introduce en cromosomas de células en división) y lentivirus (se introduce en células que no se están dividiendo), la inactivación de genes in vivo mediante la inducción de mutaciones y la inyección en espermatozoides ${ }^{(11)}$. Los animales de experimentación genéticamente modificados pueden usarse como modelos genéticamente definidos, en los que las mutaciones genéticas que predisponen o participan en el desarrollo de la enfermedad puedan controlarse para recapitular los procesos que tienen lugar en la enfermedad. Proporcionan una visión más adecuada del proceso de la enfermedad y permiten obtener mejores modelos experimentales para desarrollar y 
ensayar nuevas terapias. La transferencia de genes recombinantes a estos organismos (transgénesis), dirigidos para que se expresen en ciertos tejidos por medio de promotores específicos, permite generar proteínas recombinantes valiosas para la medicina y en la producción de alimentos.

Se pueden citar algunos ejemplos: pruebas de vacunas, banco de pruebas de drogas y medicamentos, producción de fármacos, anticuerpos, proteínas, modelos de investigación de infecciones, estudios de bases genéticas de enfermedades humanas, diseños de terapia, control de fertilidad, modelos de animales para analizar los efectos de la modulación, activación o supresión de la expresión génica, estudio del rol fisiológico de hormonas, crecimiento mayor y más rápido con la introducción de la hormona del crecimiento, y la domesticación de especies.

\section{PREOCUPACIONES ÉTICAS POR EL USO DE ANIMALES EXPERIMENTALES}

El uso de animales como modelos de enfermedades humanas se ha criticado justamente por no poder representar completamente la condición humana y obligarles a padecer condiciones deteriorantes hasta finalizar el estudio. Las pruebas de toxicidad pueden llegar a ser muy crueles pues se evalúa la toxicidad aumentando la dosis hasta producir la muerte del $50 \%$ de los animales. En estudios con fármacos, el excluir todas las fuentes de posible infección puede llevar a hacer vivir a los animales bajo condiciones excesivamente sanitarias, siendo microbiológicamente estériles.

\section{Existen otros riesgos a evaluarse:}

1. La introducción de transgénicos animales puede provocar desequilibrios en los ecosistemas, si poseen ciertas ventajas sobre especies silvestres. Peces y mariscos producidos por ingeniería genética pueden suponer riesgos adicionales ecológicos, debido a que organismos acuáticos tienen la capacidad de sobrevivir en la naturaleza, moviéndose fácilmente desde un ambiente controlado a uno natural y podrían competir con las especies nativas suplantándolas y degradar ecosistemas por el alto grado de consumo de alimentos. El salmón y la trucha, por ejemplo, son especies que al introducir la hormona de crecimiento podría desplazar a especies autóctonas ${ }^{(12)}$.

2. Riesgos en el bienestar de animales debido a dificultades en la técnica de formación de organismos genéticamente modificados. Pueden ocurrir los siguientes daños en la transgénesis: pérdida de función de un gen del huésped (debido a mutaciones, inserciones), expresión inapropiada del transgénico, exposición a proteínas producidas por el transgénico con efectos adversos, dificultades en el parto al usar fecundación in vitro y abortos espontáneos por anormalidades fetales ${ }^{(13)}$. Puede también que no se desarrolle la característica en la forma esperada.

3. En xenotrasplantes, en que se trasplantan órganos animales en humanos que tienen deteriorada su función, está la presión de usar primates como donantes de órganos y también la preocupación por la salud humana, ya que podrían transmitirse enfermedades de los animales, pudieran activarse retrovirus endógenos del animal después del trasplante con graves consecuencias para la salud pública (14). Asimismo, puede haber también rechazo crónico de órganos incluso si se previene exitosamente el rechazo agudo. Además, existe la duda de que los órganos animales, con sus características fisiológicas y metabólicas diferentes puedan efectivamente sostener la vida humana.

\section{REFLEXIÓN BIOÉTICA}

Se justifica el uso de los animales para experimentación científica por tener como fin el uso en alimentación, salvar vidas mediante terapia y liberar de sufrimiento a seres humanos, pero dicho uso impone al ser humano la responsabilidad moral de respetar al animal, no haciéndole sufrir innecesariamente, al estar trabajando con seres vivos con capacidad de sentir.

Por otra parte, para algunos bioeticistas la modificación genética en animales es inmoral en sí misma por afectar al valor intrínseco de los seres vivos. En la producción de transgénicos se da una transferencia genética que no es espontánea. Existe una intervención directa, intencional de la tecnología de la ingeniería genética, lo que los convierte en productos tecnológicos. En la polémica intervienen creencias, temas de cuestionamiento como el papel de Dios y la sacralidad de la naturaleza; dándose también luchas de poder, tal como limitar el inmenso poder de las empresas biotecnológicas y el poder ser propietario de formas de vida mediante patentes. Las preocupaciones morales y éticas son factores importantes que influencian en la aversión de las personas sobre posibles riesgos ocasionados por la producción de transgénicos ${ }^{(15)}$.

La reflexión bioética favorece el diálogo horizontal entre expertos y legos, en la medida en que se escuchen las partes y se determine exactamente los beneficios y riesgos reales de los transgénicos. También es importante el diálogo sobre la diferencia entre natural y artificial. Algunos medios y contextos clasifican lo artificial como malo de forma acrítica.

La tendencia actual en el uso de animales de experimentación es la de una visión antropocéntrica, en 
la que se reconoce al ser humano como el único ente moral. El ser humano tiene todas las prerrogativas para manipular y usar los recursos naturales a su antojo. Bajo la cultura de las transnacionales y la globalización se apuesta por una sociedad impulsada por el lucro en que el uso de los recursos de la naturaleza es ilimitado siempre que produzca beneficios. Una corriente más moderada, presente en las pautas éticas, es la de un antropocentrismo débil que se desliga de una relación con la naturaleza meramente instrumental. Se reconoce que el ser humano es capaz de transformar la naturaleza, pero esta también tiene el poder de modificar al ser humano, por lo que la relación con la naturaleza es complementaria y cualquier daño que se le inflija termina también por afectar al ser humano ${ }^{(16)}$. La relevancia moral de los organismos se encuentra en la globalidad y universalidad de las relaciones, ya que cada uno está conectado con otros organismos. Esta visión es más próxima a la cultura indígena, en que el ser humano posee vínculos con los demás seres vivos. Bajo la corriente ecocéntrica se da relevancia moral al ecosistema, la naturaleza es un ente unitario y cada organismo se define en función de sus relaciones con el medio ambiente y otros organismos ${ }^{(17)}$. Las diferentes formas de ver la relación con la naturaleza han de entrar en diálogo en la toma de decisiones.

Además, existe responsabilidad hacia generaciones futuras. Hans Jonas ha reflexionado sobre este principio, que afecta no solamente a seres humanos, sino también a todos los seres vivos, de forma que las generaciones futuras cuenten con un ambiente y biodiversidad al menos comparable al presente ${ }^{(18)}$. La responsabilidad significa reflexionar sobre las acciones a tomar teniendo en cuenta el balance entre riesgos y beneficios en el desarrollo social. Los seres humanos tienen un gran poder sobre la naturaleza gracias a la biotecnología, pues pueden alterar y manipular la vida en gran medida, lo cual requiere regulación. Éticamente hay que evitar una concepción instrumental de la naturaleza, ya que los seres vivos tienen un valor inherente en sí mismos. Esto justifica el que se establezcan límites en la actuación humana sobre la naturaleza para impeir el que se considere a los seres vivos como meros objetos susceptibles de apropiación.

Las investigaciones con animales deben someterse a un comité de ética que vele por su protección y cuidado. Su función no solamente consiste en evaluar la adecuación ética y validez científica de protocolos de investigación, sino también garantizar el trato humanitario de los animales de experimentación en bioterios. Los comités institucionales para el cuidado y uso de animales experimentales (CICUAL) deben actuar como entes independientes evitando conflictos de interés, defendiendo el bienestar del animal en el ambiente de investigación, escuchando el sentir de la sociedad y manejando los conflictos de interés potenciales entre los intereses de la sociedad, investigadores e instituciones.

Fuentes de finaciamiento: autofinanciado.

Conflictos de interés: el autor declara no tener conflictos de interés.

\section{REFERENCIAS BIBLIOGRÁFICAS}

1. Heilman RD. Drug development history, "overview," and what are GCPs? Qual Assur. 1995;4(1):75-9.

2. Zbinden G. Predictive value of animal studies in toxicology. Regul Toxicol Pharmacol. 1991;14(2):167-77.

3. National Research Council. Guide for the care and use of laboratory animals. 8th Edition. Washington, DC: National Academies Press; 2011.

4. Cardoso de Martínez C, Mrad de Osorio A, Martínez CC, Rodríguez Yunta E, Lolas Stepke F. El animal como sujeto experimental. Aspectos técnicos y éticos. Santiago de Chile: Centro Interdisciplinario de Estudios en Bioética (CIEB), Universidad de Chile; 2007.

5. Russell WMS, Burch RL. The principles of humane experimental technique. London: Methuen; 1959.

6. Marshall H. A critical and experimental essay on the circulation of the blood. London: Sherwood; 1831.

7. Departamento de Agricultura y Ganadería de Estados Unidos. Guía para el cuidado y uso de los animales de laboratorio. Washington, DC: National Academy Press; 1996.

8. Diario Oficial de las Comunidades Europeas. Directiva 2001/18/CE del Parlamento Europeo y del Consejo. 12 de marzo de 2001.

9. Gordon JW, Scangos GA, Plotkin DJ, Barbosa JA, Ruddle FH. Genetic transformation of mouse embryos by microinjection of purified DNA. Proc Natl Acad Sci U S A. 1980;77(12):7380-4.

10. Misran RP, Duncan SA. Gene targeting in the mouse: advances in introduction of transgenes into the genome by homologous recombination. Endocrine. 2002;19(3):229-38.

11. Gama Sosa MA, De Gasperi R, Elder GA. Animal transgenesis: an overview. Brain Struct Funct. 2010;214(2-3):91109.

12. Devlin RH, Biagi CA, Yesaki TY, Smailus DE, Byatt JC. Growth of domesticated transgenic fish. Nature. 2001;409(6822):781-2.
13. Van Reenen CG, Meuwissen TH, Hopster H, Oldenbroek K, Kruip TH, Blokhuis HJ. Transgenesis may affect farm animal welfare: a case for systematic risk assessment. J Anim Sci. 2001;79(7):1763-79.

14. Fishman JA, Patience C. Xenotransplantation: Infection risk revisited. Am J Transplant. 2004;4(9):1383-90.

15. Wadman M. Genetic resistance spreads to consumers. Nature. 1996;383(6601):564.

16. Velayos C. La dimensión moral del ambiente natural:¿Necesitamos una nueva ética? Granada: Editorial Comares; 1996.

17. Lovelock JE. Gaia, una nueva visión de la vida sobre la Tierra. Barcelona: Ediciones Orbis; 1985.

18. Jonas H. El Principio de Responsabilidad. Barcelona: Círculo de Lectores/Herder; 1995.

Correspondencia: Eduardo Rodríguez Yunta

Dirección: Diagonal Paraguay 265, oficina 806.

Santiago, Chile.

Teléfono: (56) 29782254

Correo electrónico:erodriguezchi@gmail.com 